\title{
Strategies to Maximize Science Data Availability for the GOES-R Series of Satellites
}

\author{
Renee Dudley ${ }^{1}$ \\ SGT, Inc., Greenbelt, Maryland,USA, renee.p.dudley@nasa.gov \\ Douglas C. Freesland ${ }^{2}$ \\ The ACS Engineering Corporation, Clarksville, MD, USA, douglas.c.freesland@nasa.gov \\ Edwin Harvie ${ }^{3}$ \\ IAI North America, Arlington, Virginia, USA, edwin.j.harvie@noaa.gov \\ Thomas Kenney ${ }^{4}$, \\ Chesapeake Aerospace, LLC, Grasonville, Maryland, USA, thomas.m.kenney@nasa.gov \\ Alexander Krimchansky, ${ }^{5}$ \\ NASA Goddard Space Flight Center, Greenbelt, Maryland, USA, alexander.krimchansky@nasa.gov \\ Seth Napora ${ }^{6}$, \\ ASRC Federal Space and Defense, Beltsville, Maryland, USA, seth.o.napora@nasa.gov \\ Chris Wheeler ${ }^{7}$ \\ NOAA Office of Satellite and Product Operations, Suitland-Silver Hill, MD, USA, chris.a.wheeler@noaa.gov \\ Tim Walsh ${ }^{8}$ \\ NOAA GOES-R Program, Greenbelt, MD, USA, tim.walsh@noaa.gov
}

\begin{abstract}
The Geostationary Operational Environmental Satellite-R Series (GOES-R) is the next generation of United States geostationary weather satellites. The GOES-R series significantly improves the detection and observation of environmental phenomena that directly affect public safety, protection of property and the economic health and prosperity of the United States and all countries within the Western Hemisphere. Given the real-time or "now-casting" nature of the GOES science gathering mission, any data outage or interruption can reduce warning times or scientific fidelity for critical weather data. GOES-R mission level requirements limit key performance product outages to a total of six hours per year to maximize science data availability. Lower level requirement only allow for 120 minutes of disruption between the spacecraft bus interface to the instruments. This requirement is met using both design features of the satellite and ground system, in addition to operational strategies.
\end{abstract}

\footnotetext{
${ }^{1}$ Deputy Mission Operations Manager, GOES-R Mission Operations Support Team, NASA GSFC.

${ }^{2}$ Guidance Navigation and Controls, GOES-R Flight, NASA GSFC.

${ }^{3}$ GOES-R Team Lead, GOES-R Mission Operations Support Team, NOAA NSOF.

${ }^{4}$ Contingency Manager, GOES-R Mission Operations Support Team, NOAA NSOF.

${ }^{5}$ System Manager and Chief Architect, GOES-R Flight, NASA GSFC.

${ }^{6}$ Spacecraft Navigation Engineer, GOES-R Mission Operations Support Team, NOAA NSOF.

${ }^{7}$ Mission Operations Manager, GOES-R Mission Operations Support Team, NOAA NSOF.

${ }^{8}$ System Program Director (Acting), GOES-R Program, NASA GSFC.
} 


\section{Introduction}

The Geostationary Operational Environmental Satellite-R (GOES-R) series is the next generation of the United States' geostationary weather satellites. The GOES-R series significantly improves the detection and observation of environmental phenomena that directly affect public safety, protection of property and the economic health and prosperity of the United States and all countries within the Western Hemisphere. The GOES-R series satellites include six advanced instruments that provide imaging with increased spatial resolution and faster coverage for more accurate forecasts, real-time mapping of lightning activity, and improved monitoring of solar activity. The first GOES-R series satellite - designated GOES-16 on-orbit - was launched in November 2016 and now serves as the operational GOESEast mission. The second in the series - designated as GOES-17 on-orbit - was launched in March 2018 and is currently undergoing pre-operational testing before becoming the operational GOES-West mission.

Given the real-time or "now-casting" nature of the GOES science gathering mission, any data outage or interruption can reduce warning times or scientific fidelity for critical weather data. Using a four day cycle of east/west, north/south and momentum adjust operations using low-force thrusters allows all six GOES-R instruments to operate continuously through thruster related operations. GOES-16 in-flight test results have demonstrated the feasibility of this operational approach to maximize data availability while introducing the potential of more autonomous operations. Other design features significantly maximize the availability of science data over previous GOES spacecraft. This includes the use of onboard GPS for orbit determination, the thermal design of the ABI (which eliminates the need for yaw maneuvers), dither calibrations to reduce downtime created by IMU calibrations, autonomous fault detection and correction, and the use of SpaceWire and the GOES-R Reliable Data Delivery Protocol. [1]

This paper reviews some of the design features and the operational approaches used to maximize science data availability, meeting the requirement that limits scheduled disruption to the interface between the spacecraft bus and the instruments to 120 minutes per year.

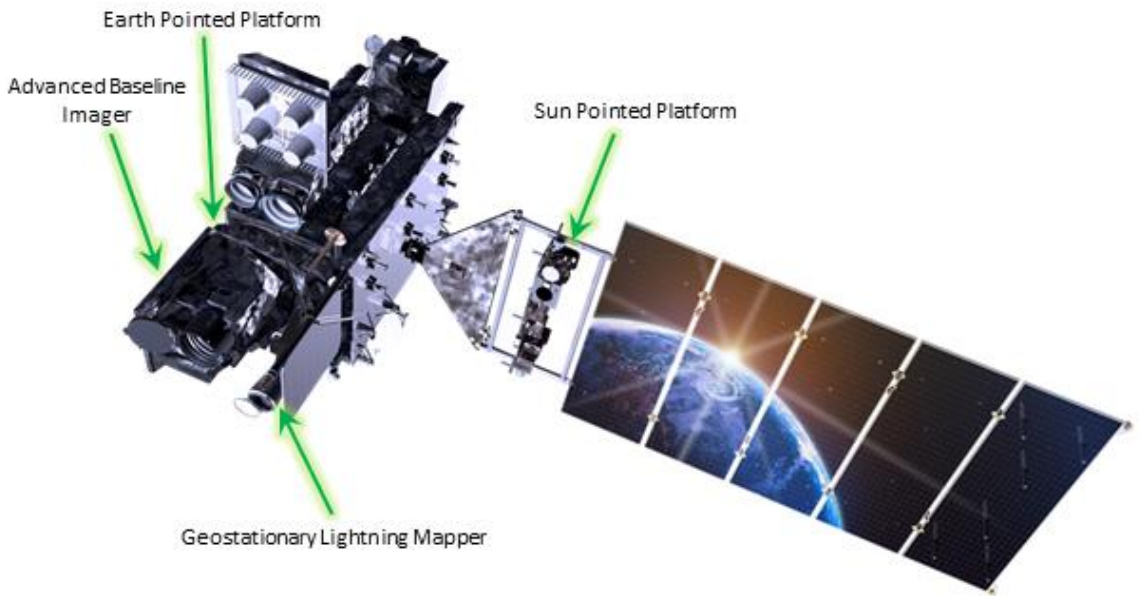

Fig. 1 GOES-R Series Spacecraft.

\section{Thruster Design and Maneuver Strategy Allow to "Operate Through" Stationkeeping}

The GOES-R series of satellites were designed to continuously generate scientific products throughout their lifetimes with minimal downtime, limited to a total of six hours per year. Of this six hours, only two hours are allocated to downtime relating to the interface between the spacecraft bus and the instruments. To fulfill this requirement, GOES-R was designed to "operate through", i.e. to maintain the spacecraft's pointing accuracy during thruster activities, maximizing the availability of science data. The design led to the incorporation of low-thrust, highefficiency thrusters for stationkeeping and momentum adjust operations. This approach has resulted in small, frequent maneuvers to preserve its orbital position and manage momentum [2]. By comparison, the previous GOES-N Series 
allowed for up to two ten minute outages per day. These outages were used for daily momentum adjusts (MA), east/west stationkeepings (EWSK) that occurred approximately four times per year, and other spacecraft/instrument related maintenance activities. Additionally, there was allowance for up to six hour outages for annual north/south stationkeeping (NSSK), and twenty four hour outages for bi-annual yaw maneuvers.

To meet operate-through requirements, Lockheed Martin's thruster selection and placement allow the vehicle to continue science observations during stationkeeping maneuvers (MA, EWSK, and NSSK). Because of thruster locations, orbital maintenance maneuvers are accomplished without any requirement to slew the vehicle. In addition, small force thrusters are used to reduce attitude disturbances during thruster related activities. More specifically, $0.10 \mathrm{~N}$ low thrust rocket engine assemblies (LTRs) perform MA and EWSK while $0.22 \mathrm{~N}$ Arcjets are used for NSSK. The small force levels of these thrusters allow the instruments to meet imaging requirements during maneuvers. The figure below demonstrates a large improvement in attitude error about each body axis between the GOES-N and GOES-R series during a MA maneuver.

Attitude Error During a Maneuver GOES-N Series vs GOES-R Series

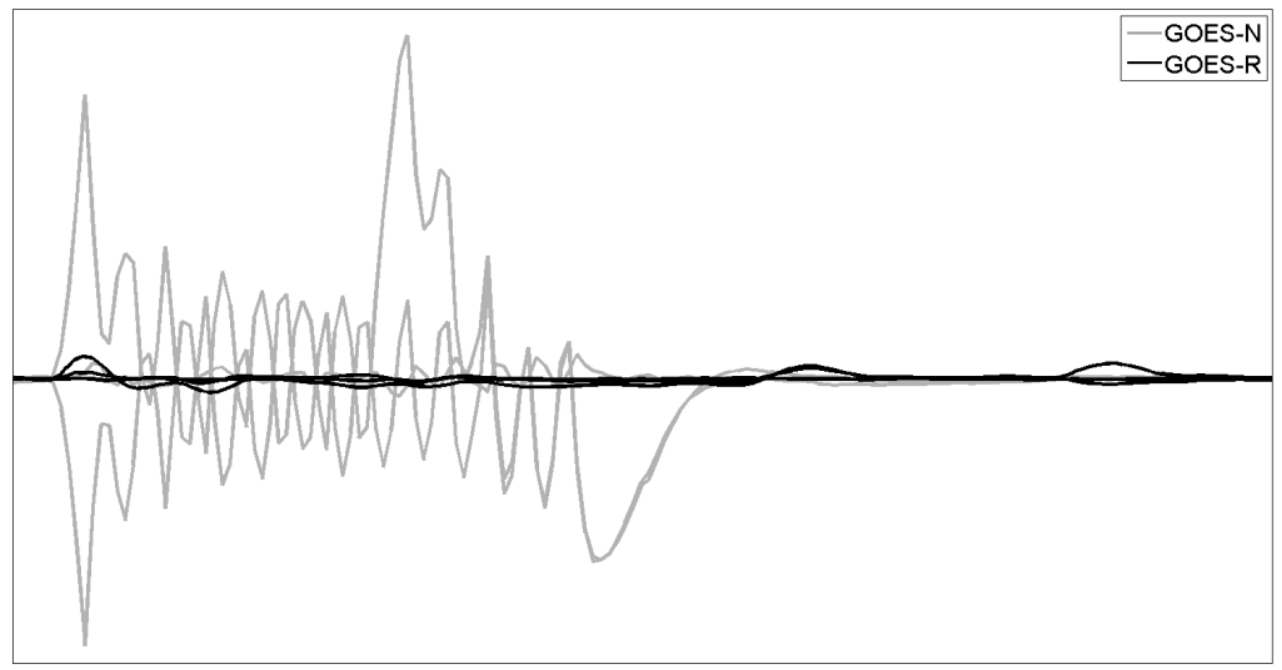

Fig. 2 Relative Attitude Error of GOES-N versus GOES-R

The lower thrust produced by the GOES-R series spacecraft thrusters results in more frequent orbital maintenance activities compared to the GOES-N series. As illustrated below, the GOES-R series uses a four day maneuver cadence to maintain its orbit box of +/-0.1 degrees. EWSK maneuvers are used to augment the spacecraft's longitude and eccentricity while NSSK and standalone MA maneuvers are designed to change the vehicle's inclination. MA maneuvers around the NSSK are used to manage the momentum buildup generated by the NSSK.

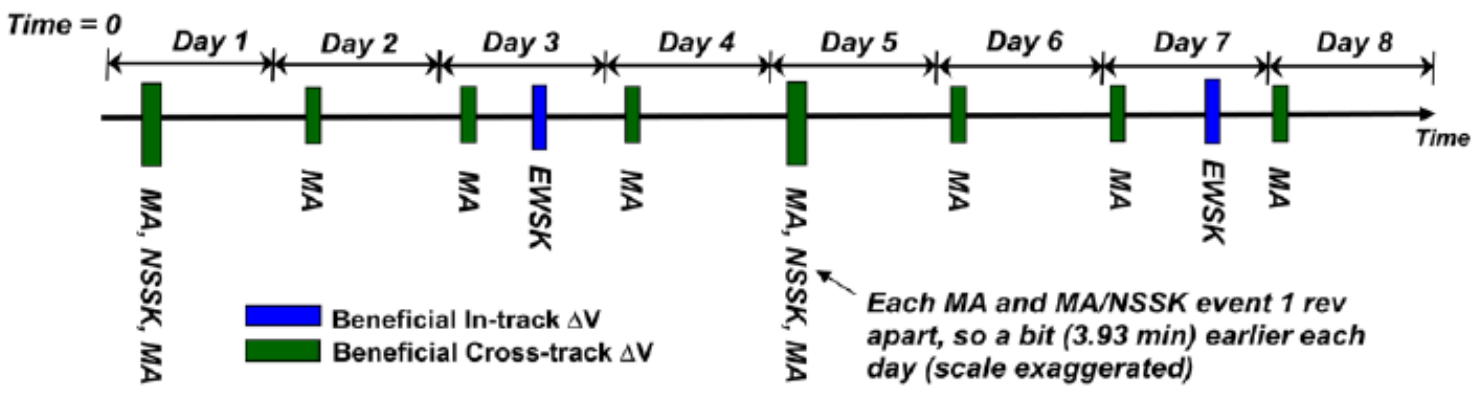

Fig. 3 GOES-R Series Maneuver Strategy 
The GOES-R series ground system navigation software is used to plan this complex cadence of maneuvers. The operations team has extensively automated the navigation software to reconstruct executed maneuvers, perform orbit determination, and plan maneuvers for up to seven days. Once the maneuver plan has been generated and reviewed, it is compiled into an Absolute Time Sequence (ATS) for upload and autonomous execution by the spacecraft at specified times. Additionally, the ATS contains the commanding required for the spacecraft bus and instrument operations for the duration of the plan. This capability permits seven days of autonomous operations of the spacecraft with no ground interaction, in the event of a significant ground related anomaly that would prevent commanding to the spacecraft. The scheduled maneuvers ensure the spacecraft maintains its momentum state and orbital slot while the spacecraft bus and instrument commands ensure uninterrupted science data.

\section{Use of GPS for Orbit Determination to "Operate Through" Stationkeeping Maneuvers}

More than a decade before the first GOES-R launched in November 2016, system architects were focused on requirements that increased operational availability while simplifying mission operations [3]. One such pioneering technology was the use of a Global Positioning System Receiver (GPSR) at GEO. Not only did this eliminate the need for ranging, data processing, and daily ephemeris uploads, but it allowed for maintaining a tighter orbital slot \pm 0.05 degree. The primary driver, however, was that it achieved stringent INR requirements prior to, during and after stationkeeping maneuvers. This operate through capability eliminates interruption of science data for routine thruster based events. Orbital position and velocity knowledge requirements are summarized in Table I.

Table I GOES-R Orbital Position and Velocity
Knowledge Requirements
\begin{tabular}{lrc}
\hline \multicolumn{3}{c}{ Positon } \\
\hline In-Track & $75 \mathrm{~m} 3 \sigma$ & $6 \mathrm{~cm} / \mathrm{sec} 3 \sigma$ \\
Cross-Track & $75 \mathrm{~m} 3 \sigma$ & $6 \mathrm{~cm} / \mathrm{sec} 3 \sigma$ \\
Radial & $100 \mathrm{~m} 3 \sigma$ & $6 \mathrm{~cm} / \mathrm{sec} 3 \sigma$ \\
\hline
\end{tabular}

The 12 channel General Dynamics Viceroy IV GPSR was selected for the GOES-R series; the first use of a civilian frequency GPSR at GEO, providing a dramatic step forward in autonomous orbit determination [4,5]. It was coupled with a unique L1 antenna built by Lockheed Martin Space Systems designed for side lobe tracking with a main lobe gain of $\sim 11 \mathrm{~dB}$ at 22 degrees off nadir. The ability to use the side lobes vastly increases the availability of GPS signals along with the accuracy achievable. On average, GOES-16 incorporates over 11 GPS satellites in its solution with no dropouts. A 50 hour span of GPSR data was collected, downlinked and processed offline using an Extended Kalman Filter (EKF) process. Results are shown in Fig. and demonstrate the ability to operate through two North/South stationkeeping maneuvers of $\sim 2 \mathrm{~cm} / \mathrm{sec} \sim 24$ hours apart.

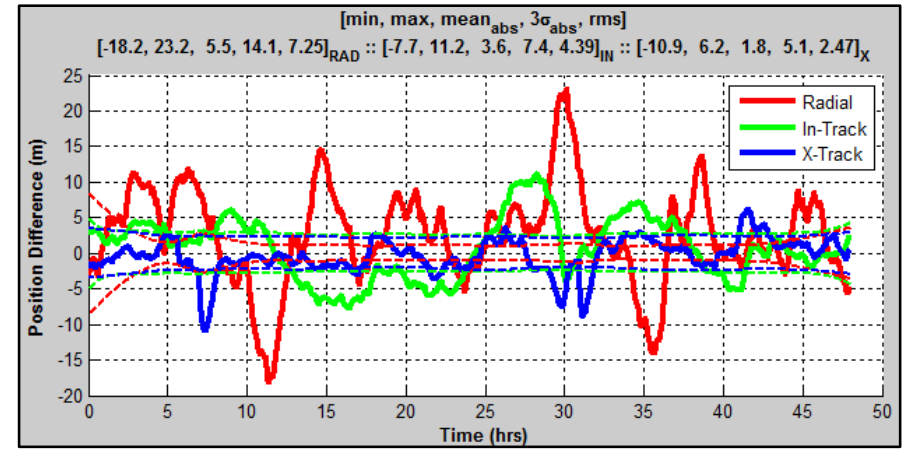

Fig. 4 GOES-16 GPSR position error as difference with ground EKF solution. 


\section{Robust Thermal Design Eliminates Need for Yaw Maneuvers}

The previous series of GOES spacecraft required twice-yearly yaw maneuvers to accommodate thermal constraints on the instruments. These yaw maneuvers impacted data products for up to twenty-four hours following a yaw maneuver execution. The GOES-R team set out to design a spacecraft that would not require seasonal yaw inversion operations to address thermal concerns and eliminate the significant loss of science data associated with this type of maneuver. The Advanced Baseline Imager (ABI) and Global Lightning Mapper (GLM) are located on the Earth Pointed Platform (EPP), but in view of the solar array (SA) and sun pointed platform (SPP), both of which create a backload on their respective radiators. By oversizing their radiators, these instruments can meet radiometric requirements without seasonal yaw maneuvers to account for changes in the sun angle.

The primary instrument on the GOES-R series satellites is the ABI. This instrument is located on the thermally isolated EPP and has demanding thermal constraints. The ABI design uses a mechanical cryocooler to cool its focal plane arrays. A scan shroud assembly protects the internal instrument structure from direct solar loading through the optical port during the times in the orbit when solar energy enters the internal instrument cavity. Additionally, loop heat pipes (LHP) interface with an oversized $\left(2.3 \mathrm{~m}^{2}\right)$ radiator that work together to transfer excess thermal energy from the instrument to space. The radiator creates a sink with an effective temperature of $205 \mathrm{~K}$ when exposed to the sun at a +/-24 degree angle. The GOES-R design is capable of rejecting $330 \mathrm{~W}$ of thermal energy, although in the worst case only $290 \mathrm{~W}$ needs to be radiated. The oversized radiator provides a $40 \mathrm{~W}$ margin for energy transfer, allowing the $\mathrm{ABI}$ to operate without requiring a seasonal yaw maneuver. The larger optical surface reflectors (OSR) on the radiators will degrade over the lifetime of the spacecraft, but the $40 \mathrm{~W}$ margin is large enough to account for the associated decrease in efficiency.

The GLM is also located on the EPP and had similarly challenging requirements for thermal design. GLM's radiator is sized at about $2 \mathrm{~m}^{2}$, creating a heat sink with an effective temperature of $247 \mathrm{~K}$ and capable of rejecting $240 \mathrm{~W}$ of thermal energy. Originally, it was estimated that the GLM would require approximately 165W, but upon completion of the GLM design the actual need was $35 \mathrm{~W}$ less. This gives the GLM greater than $75 \mathrm{~W}$ margin, allowing the instrument to fly without seasonal considerations or yaw flip. This margin is more than enough to account for the decreased efficiency of the OSRs over the life of the spacecraft.

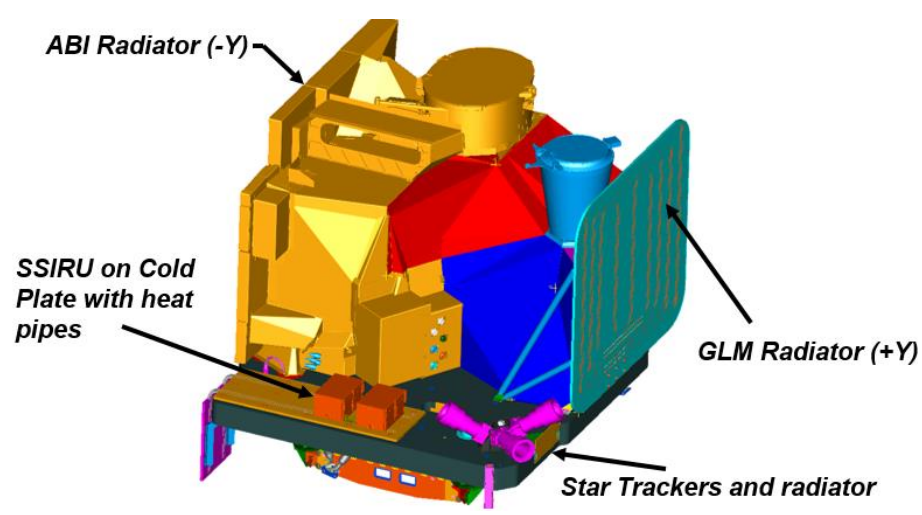

Fig. 5 Earth Pointed Platform.

\section{Use of Dither Calibrations to Reduce Downtime from IMU Calibration Maneuvers}

The GOES-R series spacecraft use the Northrop Grumman Scalable Space Inertial Reference Unit (SSIRU) Inertial Measurement Unit (IMU) for attitude determination. The IMU rate data is used onboard for ABI pointing control and on the ground for ground image navigation. As gyro scale factor errors increase over the operational life, ABI pointing and Image Navigation and Registration (INR) will suffer, particularly during thruster operations. Lockheed Martin 
determined during GOES-16 Post Launch Testing (PLT) that a scale factor recalibration should be performed every two to five years, based on the drift observed between the beginning and end of PLT. These recalibrations involve slewing the spacecraft 3.5 degrees about each gyro axis, which creates a loss of science data for approximately one hour and can impact INR (degrading science data) for up to thirty hours.

An innovative alternative technique for IMU scale factor determination called "dither calibration" can be performed with all instruments operating nominally, with no loss of data. The dither calibration technique involves a series of very small angle slews over a long duration to derive IMU scale factor errors statistically while not exceeding science data pointing specification. After dither calibration was successfully tested during GOES-16 PLT, the GOESR Flight Project recommended these calibrations be performed twice a year, near each equinox, in order to trend scale factor drift. This provides a non-invasive, though less accurate estimate of scale factor. This will show if scale factor has drifted faster or further than expected, requiring the small slew recalibration sooner than five years, or conversely, if it indicates lower than expected drift, the recalibrations could be postponed or eliminated, minimizing operational downtime and data loss. [6]

Although dither is mostly non-invasive, dither can trigger false lightning events on the Global Lightning Mapper (GLM) if performed during daylight hours due to the tuning of the GLM event detection algorithms. For this reason, dither calibrations are performed around midnight. At midnight on the equinoxes, the dither excitation axis (s/c yaw) is nearly aligned with the sun vector, which also minimizes yaw-axis dither coupling into Sun Pointed Platform (SPP) pointing.

For GOES-17, Lockheed Martin has augmented the Guidance, Navigation, and Control (GN\&C) flight software to include an onboard dither generator. This will be tested during GOES-17 PLT, with the goal of further reducing the impact of dither so that the calibration can be performed at any time of day or day of year. The lower amplitude will come at a "cost" of longer duration of excitation. If findings from GOES-17 PLT can be transferred to GOES-16, scheduling of these calibrations for GOES-16 could be relaxed to avoid to midnight staffing.

\section{Autonomous Fault Detection and Correction to Prevent Data Loss}

Early in the development of the GOES-R series spacecraft, Lockheed Martin determined that to meet data availability requirements for this mission, it would be necessary to have the capability to autonomously identify and resolve as many faults as possible. To accomplish this objective, the fault management design used a hybrid approach, comprised of three distinct onboard systems. The first is an inline fault detection and correction scheme. The second uses software objects representing the individual elements of the flight hardware and software system. Finally, there is the combination of telemetry monitors (TMON) and relative time sequence (RTS) stored command loads. Together, these three systems provide for monitoring and acting on more than 2200 distinct faults.

The inline detectors came from heritage code or code that was already in advanced development prior to deciding the final GOES-R series fault management design. These detectors check for out of limits values during the execution of the nominal subsystem flight code. The limits and tolerances on these detections are parameterized. However, their design does not follow the object template. These detectors were normally designed to execute a predefined response to an out of limits condition, however, most have been interfaced to the object based system.

The software objects are a new element for the Lockheed Martin spacecraft and implemented on the GOES-R series for the first time. The object scheme implements three tiers of fault monitoring: component, domain, and system. The first level is the component level. These are coded objects that represent specific hardware instances. These objects respond to the commanded state of the corresponding hardware and are capable of adjusting the expected outputs in the telemetry. When a monitored output of an object is out of defined limits, the output is considered to be in error. When error persistence exceeds a configurable interval, the output is considered to be in a fault condition. The model allows for different responses to be defined when an error occurs and when a fault occurs. When it is determined that a component is in fault the error can propagate up to the next level, the domain. There are several domain monitors that correspond to functions of the various subsystems (e.g. the Attitude Control Domain Monitor). The domain level is where most component swaps occur. Should the domain level response fail to correct the issue, the fault will propagate to the final tier, System Level Fault Management. The system level responses to faults do interrupt normal operations and can be divided into two levels of response: Safe Hold Entry and Heartbeat Termination. 
This tiered system of detections and responses allows for a detected error condition to be addressed at the lowest level possible while also accommodating elevated responses if the initial response proves ineffective. An example of this would be the voltage monitors in the six reaction wheels (RWA). For each RWA there is an object that includes a monitor on the $5 \mathrm{~V}$ supply, which functions differently based on the commanded power state of the RWA. When the RWA is commanded on, if the $5 \mathrm{~V}$ output of the RWA falls outside the limits, the initial error response is to command the wheel to its expected powered state, ON. Alternatively, if the RWA has been commanded off, if the 5V output rises above a limit, the initial error response is also to command the wheel to its expected powered state, in this case OFF. If the error response fails to correct the problem and the fault persistence limit is reached the RWA will be marked failed. When a single RWA is marked as failed an object in the Attitude Control Domain then responds and alters the attitude control suite to use a configuration with the remaining 5 RWA.

Finally, there is the system of the Telemetry Monitors (TMON) and Real Time Sequences (RTSs). TMONS and RTSs are able to monitor all telemetry data generated onboard, not just the values that are being downlinked in the currently selected telemetry configuration. TMONs are currently being used for short term fault management functions (e.g. over-temperature monitoring during frangibolt firings). This element of the system provides the best path for adding new elements to the fault management system as it does not require a flight software patch to implement. Recently a TMON/RTS combination was able to provide an "operate through" capability to an apparent electro-static discharge (ESD) upset event on GOES-16 that would have otherwise produced an extended outage of solar observing instruments.

The complexity of operations and potential for interaction associated with the number of fault monitors on GOES$\mathrm{R}$ led to concern during the system development. A special test program, designed around worst case scenarios, was created to demonstrate that the system would be robust. After launch, some tuning of the fault monitors was required to reduce the instances of false alarms. To date, no false alarm has led to discontinuities in operation or loss of data.

\section{GOES-R Reliable Data Delivery Protocol Reduces “Lost” Data}

Data loss starts at the data transfers between the instruments and spacecraft, and requires error detection and correction (EDAC) at the packet level. SpaceWire was selected for the science data transfer as well as command and telemetry data for the GOES-R series instruments. Unfortunately, the SpaceWire standard [7] does not specify a protocol for reliable data delivery. Because the GOES-R Program required a reliable data delivery protocol for all data transferred over SpaceWire, the GOES-R Reliable Data Delivery Protocol (GRDDP) was developed in-house [8] to provide a means of reliably delivering data among various on board data sources and sinks. Additionally, the spacecraft design was required to support redundant SpaceWire links for each instrument side, as well as to route the fewest number of connections through a Slip Ring Assembly necessary to support solar pointing instruments. This design redundancy significantly minimizes, if not eliminates, loss of science data.

The GRDDP adds capability to the SpaceWire link through multiplexed logical connections, reliable delivery, missing packet detection, and out-of-sequence packet reordering. Reliable delivery includes detection of lost packets, duplicate packets, out of sequence packets, and provides corrupted data recovery. It also provides additional error detection beyond the SpaceWire physical layer utilizing cyclical redundancy checks (CRCs), packet sequence numbers, positive acknowledgement, and timeouts to detect lost or duplicated data packets. Acknowledgements are required for all packets - if one is not received within a specified timeout, the source node is required to retransmit. GRDDP packets are also bounded in size, which can be set in the hardware implementation.

This feature is very useful when the spacecraft experiences "lost" instrument packet data due to radiation effects. The retransmit provides excellent protection from electrostatic discharge (ESD) in the SpaceWire cable. Since the launch of the initial GOES-R series satellite (GOES-16), the spacecraft has not lost a single packet on the SpaceWire

link due to radiation or ESD in the SpaceWire cables. It has also provided an accurate accounting of the communication links between the instruments and spacecraft. 


\section{Conclusion}

The increased data continuity requirements of the GOES-R series missions represent a watershed in geostationary meteorological observations available to NOAA and the National Weather Service. Data outage allocations have been reduced from almost 150 hours per year for the GOES-N/O/P series to less than 6 hours per year for the GOES-R series. In practice, flight experience with GOES-16 since it became operational as GOES-East in June 2017 has shown that no science data has yet been lost due to satellite operational activity.

The sophisticated design of the spacecraft and instruments coupled with innovative operational strategies has proven that it is possible to successfully operate with minimal loss of science data. Soon, GOES-17 will become GOES-West, to provide full coverage of the Western Hemisphere, so that that this state of the art mission is able to provide scientists with all of the data they need to help protect the property and the economic health and prosperity of the United States and all countries within the Western Hemisphere.

\section{Acknowledgments}

The authors thank the entire GOES-R team for their exceptional support of this mission. Special thanks to Doug Freesland for his invaluable help.

\section{References}

[1] Freesland, D., Chu D., Reth, A, Krimchansky, A., Donnelly, M., Comeyne, G., "Advancing the Next Generation GOES-R Operational Availability," 41 st Annual AAS Guidance and Control Conference, AAS 05-006, Breckenridge, CO, USA, February 2005.

[2] Chapel, J., Stancliffe, D., Bevacqua, T., Winkler, S., Clapp, B., Rood, T., Freesland, D., Reth, A., Early, D., Walsh, T., Krimchansky, A, "In-Flight Guidance, Navigation, and Control Performance Results for the GOES-16 Spacecraft," $10^{\text {th }}$ International ESA Conference on Guidance, Navigation \& Control Systems, Salzburg, Austria, June 2017.

[3] Freesland, D., Chu D., Reth, A, Krimchansky, A., Donnelly, M., Comeyne, G., "Advancing the Next Generation GOES-R Operational Availability," 41st Annual AAS Guidance and Control Conference, AAS 05-006, Breckenridge, CO, USA, February 2005.

[4] Winkler, S., Ramsey, G., Frey, C., Chapel, J., Chu, D., Freesland, D., Krimchansky, A., Concha, M., "GPS Receiver On-Orbit Performance for the GOES-R Spacecraft," 10th International ESA Conference on Guidance, Navigation \& Control Systems, Salzburg, Austria, June 2017.

[5] Ashman, B., Bauer, F., Parker, J., Donaldson, J., "GPS Operations in High Earth Orbit: Recent Experiences and Future Opportunities," 15th International Conference on Space Operations, Marseille, France, June 2018.

[6] Reth, A.D., Carter, D.R., Freesland, D.C, and Krimchansky, A., "Increased Operational Availability and Simplified Operations Using Dither Gyro Scale Factor Calibration," AIAA SpaceOps Conference (to be published).

[7] European Cooperation for Space Standardization, "Space Engineering: SpaceWire Protocol Identification," ECSS-E-ST-5051C, 5 February 2010.

[8] Geostationary Operational Environmental Satellite (GOES) GOES-R Series, “GOES-R Reliable Data Delivery Protocol (GRDDP)”, NASA 417-R-RPT-0050, 1 July, 2005. 